\title{
Características de pacientes hospitalizados con COVID-19 en la red asistencial La Libertad-EsSalud, 2020
}

Marco Antonio Alfaro Angulo* 1,a ; Mónica Rosaura Rivero Villegas 1,b; Víctor Andrés Sánchez Reyna 2,c

\section{RESUMEN}

Objetivo: Describir las características de los pacientes hospitalizados con COVID-19 en la red asistencial La Libertad-EsSalud, 2020. Materiales y métodos: Estudio descriptivo. Los datos se recolectaron de las fichas clínico-epidemiológicas y de las historias clínicas de los pacientes que se hospitalizaron entre el 15 de marzo y el 17 de agosto del 2020 . Las variables consideradas en la investigación fueron edad, sexo, comorbilidad, estancia hospitalaria, uso de ventilación mecánica, días con ventilación mecánica, fallecidos y pacientes de alta. Se calcularon frecuencias absolutas y relativas, y el valor de $p$. Resultados: El estudio incluyó a 2093 pacientes hospitalizados con COVID-19, el promedio de edad fue 58 años, y el 63,21 \% eran hombres. El 39,99\% tenía comorbilidades. La media de tiempo de hospitalización fue 8 días. El 9,79 \% de los pacientes ingresó a la unidad de cuidados intensivos y permaneció por 20,40 días, en promedio. El 9,56 \% requirió ventilación mecánica. Los fallecidos fueron el 40,28 \% de todos los pacientes; y el 54,50 \% de los enfermos que requirieron ventilación mecánica murieron. El 49,74\% de pacientes fue dado de alta.

Conclusiones: El mayor número de pacientes era de sexo masculino. La edad media de los participantes fue 58 años. Las principales comorbilidades fueron hipertensión arterial, diabetes y obesidad. El tiempo promedio de hospitalización fue 8,02 días. El $9,79 \%$ de los pacientes estuvieron en la unidad de cuidados intensivos; y el 9,56 \% requirió ventilación mecánica. El 40,28 \% de los enfermos fallecieron, y de los pacientes con ventilación mecánica, murieron el 54,50 \%. Los hospitalizados que salieron de alta fueron el 49,74\%.

Palabras clave: Pacientes; Hospitalizados; COVID-19 (Fuente: DeCS BIREME).

\section{Characteristics of patients hospitalized for COVID-19 in the La Libertad-EsSalud healthcare network, 2020}

\section{ABSTRACT}

Objective: To describe the characteristics of patients hospitalized for COVID-19 in the La Libertad-EsSalud healthcare network, 2020.

Materials and methods: A descriptive study. The data was collected from the clinical epidemiological records and medical records of patients hospitalized from March 15 to August 17, 2020. The research variables were age, sex, comorbidity, hospital stay, use of mechanical ventilation, number of days on mechanical ventilation, number of deaths and number of discharged patients. Absolute and relative frequencies and the $p$ value were calculated.

Results: The study population consisted of 2,093 patients hospitalized for COVID-19, whose average age was 58 years. Sixtyone point two three percent (61.23\%) were males, $39.99 \%$ had comorbidities, the mean hospital stay was 8 days, $9.79 \%$ were admitted to the ICU and spent 20.40 days on average, $9.56 \%$ required mechanical ventilation, $40.28 \%$ of the total number of patients died, $54.50 \%$ of those who received mechanical ventilation died, and $49.74 \%$ were discharged.

Conclusions: The average age of the patients was 58 years, most of whom were males. The main comorbidities were high blood pressure, diabetes and obesity. The average hospital stay was 8.02 days, $9.79 \%$ of the patients were admitted to the ICU, $9.56 \%$ required mechanical ventilation, $40.28 \%$ died, $54.50 \%$ of those who received mechanical ventilation died, and $49.74 \%$ were discharged.

Keywords: Patients; Hospitalized; Coronavirus infections (Source: MeSH NLM).

1 Red Asistencial La Libertad - EsSalud. Trujillo, Perú.

2 Hospital Especializado Víctor Lazarte Echegaray- EsSalud. Trujillo, Perú.

a Médico Cirujano, Especialista en Enfermedades Infecciosas y Tropicales.

b Médico Cirujano.

c Médico Cirujano, Especialista en Pediatría.

*Autor corresponsal. 


\section{INTRODUCCIÓN}

Desde que se detectó la COVID-19 en China ${ }^{(1,2)}$, la enfermedad se ha convertido en una pandemia. En el Perú, la epidemia se inició en marzo del año 2020 y se ha propagado a todo el país: al 16 de marzo de 2021, se han notificado 1407963 casos en el país, y 48414 en La Libertad ${ }^{(3,4)}$. En este departamento, la propagación de la enfermedad ha afectado a la población asegurada: de 18155 casos, 4122 pacientes fueron hospitalizados y 1511 han fallecido en la fecha indicada ${ }^{(5)}$.

La sintomatología se inicia luego del período de incubación de la enfermedad, que en la mayoría de los casos es de 5 días ${ }^{(6,7)}$. El diagnóstico se hace con criterios epidemiológicos y características clínicas, y se confirma con la prueba de reacción en cadena de la polimerasa ${ }^{(8)}$. La mayoría de las infecciones se autolimitan, pero los cuadros más graves ocurren en personas mayores de 60 años o con enfermedades subyacentes ${ }^{(9)}$, y requerirán hospitalización.

En el país, los casos se han clasificado en leves, moderados y graves ${ }^{(10)}$. Los casos moderados deben recibir el tratamiento en un hospital con las condiciones adecuadas para el aislamiento. En los casos graves o críticos, la enfermedad progresa rápidamente con disfunción o incluso falla de más de dos órganos, lo que pone en peligro la vida del paciente, por lo que debe ser admitido en una unidad de cuidados intensivos $(\mathrm{UCl})$ lo antes posible ${ }^{(8,10-13)}$.

En el curso de la epidemia de COVID-19, es importante conocer la información de las hospitalizaciones por esta enfermedad, tanto las características de los pacientes como el uso de los servicios, para mejorar la planificación e implementar estrategias de prevención en la comunidad y optimizar la atención de los pacientes. Por ello, el objetivo de esta investigación es describir a los pacientes hospitalizados (edad, sexo y comorbilidades), los servicios hospitalarios, la estancia hospitalaria, los pacientes internados en la unidad de cuidados intensivos (con ventilación mecánica), los fallecidos $\mathrm{y}$ los pacientes que salieron de alta.

\section{MATERIALES Y MÉTODOS}

\section{Diseño y población de estudio:}

Estudio descriptivo desarrollado en la red asistencial La Libertad de EsSalud, ubicada en el departamento del mismo nombre, que cuenta con 555 camas de hospitalización distribuidas en 12 establecimientos de salud. Los datos de los pacientes se recolectaron de las fichas clínico-epidemiológicas de los pacientes con COVID-19 y de la revisión de historias clínicas. La confidencialidad y el anonimato de las personas fueron respetados. Se denominó hospitalizado a la persona que permaneció en un centro de salud al menos 24 horas y con enfermedad confirmada por prueba de laboratorio, reacción en cadena a la polimerasa en tiempo real o prueba rápida, entre el 15 de marzo y el 17 de agosto del 2020. Las comorbilidades se seleccionaron de acuerdo con estudios previos. Las enfermedades cardiovasculares incluyeron cardiopatía coronaria, insuficiencia cardíaca, arritmias, valvulopatías y arteriopatía periférica ${ }^{(14,15)}$.

\section{Variables y mediciones}

Las variables de estudio fueron edad, sexo, comorbilidades, estancia hospitalaria, uso de ventilación mecánica, días con ventilación mecánica, los pacientes fallecidos y los que salieron de alta. Todas las variables se expresaron en porcentajes.

\section{Análisis estadístico}

Se calcularon frecuencias absolutas y relativas, y el valor de $p$.

\section{Consideraciones éticas}

El trabajo de investigación fue aprobado por el Comité de Investigación y de Ética del Hospital Víctor Lazarte Echegaray.

\section{RESULTADOS}

Se incluyeron 2093 hospitalizados con COVID-19. La mayoría de los pacientes tenía de 50 años a más (Figura 1), el promedio de edad fue 58 años (rango de 2 a 98 años) y el $63,21 \%$ eran hombres.

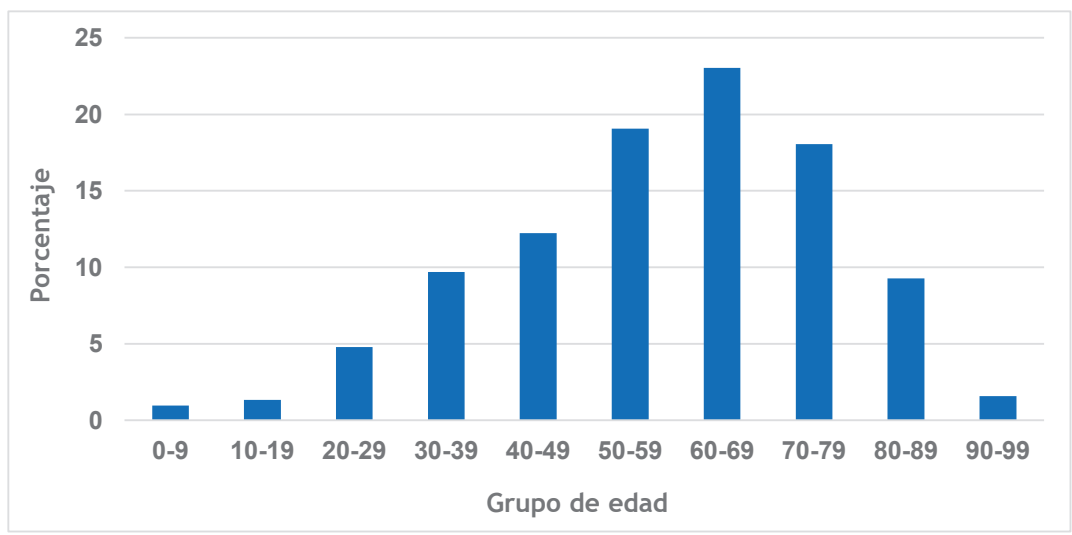

Figura 1. Distribución de los pacientes hospitalizados con COVID-19 por grupo etario, del 15 de marzo al 17 de agosto del 2020 
El $39,99 \%$ de los pacientes tenían comorbilidades. La hipertensión arterial se documentó en el 16,24 \% y fue la más común en las personas de 65 años a más $(p=0,000)$; el $11,90 \%$ sufría de diabetes mellitus, sobre todo en el grupo ya mencionado $(p=0,000)$. La obesidad se presentó en el 7,02\%, con mayor frecuencia en el grupo de 18 a 49 años $(p=0,000)$. El $1,9 \%$ tenía enfermedad cardiovascular, mientras que el cáncer, asma, enfermedad renal crónica, cirrosis y tabaquismo aparecieron en menos del $1,10 \%$ de los pacientes (Tabla 1).

Tabla 1. Asociación entre el sexo y el destino final de los pacientes atendidos por COVID-19 en el Servicio de Emergencia en el HMC

\begin{tabular}{|c|c|c|c|c|c|c|c|}
\hline \multirow[b]{2}{*}{ Comorbilidad } & & \multicolumn{5}{|c|}{ Grupo etario } & \multirow{2}{*}{$\begin{array}{c}\text { Valor } \\
p\end{array}$} \\
\hline & & $\begin{array}{l}\text { Total } \\
2093\end{array}$ & $\begin{array}{l}<18 \\
(n=44)\end{array}$ & $\begin{array}{c}18-49 \\
(n=563)\end{array}$ & $\begin{array}{c}50-64 \\
(n=639)\end{array}$ & $\begin{array}{c}\Rightarrow>65 \\
(n=847)\end{array}$ & \\
\hline \multirow[t]{2}{*}{ Alguna comorbilidad } & N. ${ }^{\circ}$ & 837 & 7 & 137 & 270 & 423 & 0,000 \\
\hline & $\%$ & 39,99 & 15,91 & 24,33 & 42,25 & 49,94 & \\
\hline \multirow[t]{2}{*}{ Ninguna comorbilidad } & N. ${ }^{\circ}$ & 1256 & 37 & 426 & 369 & 424 & 0,000 \\
\hline & $\%$ & 60,01 & 84,09 & 75,67 & 57,75 & 50,06 & \\
\hline \multirow[t]{2}{*}{ Hipertensión arterial } & N. ${ }^{\circ}$ & 340 & 2 & 12 & 102 & 224 & 0,000 \\
\hline & $\%$ & 16,24 & 4,55 & 2,13 & 15,96 & 26,45 & \\
\hline \multirow[t]{2}{*}{ Diabetes mellitus } & N. ${ }^{\circ}$ & 249 & 0 & 42 & 90 & 117 & 0,000 \\
\hline & $\%$ & 11,90 & 0 & 7,46 & 14,08 & 13,81 & \\
\hline \multirow[t]{2}{*}{ Obesidad } & N. ${ }^{\circ}$ & 147 & 1 & 66 & 61 & 19 & 0,000 \\
\hline & $\%$ & 7,02 & 2,27 & 11,72 & 15,65 & 2,24 & \\
\hline \multirow[t]{2}{*}{ Enfermedad cardiovascular } & N. ${ }^{\circ}$ & 40 & 0 & 1 & 4 & 35 & 0,000 \\
\hline & $\%$ & 1,91 & 0 & 0,18 & 0,63 & 4,13 & \\
\hline \multirow[t]{2}{*}{ Asma } & N. ${ }^{\circ}$ & 22 & 1 & 11 & 5 & 5 & 0,065 \\
\hline & $\%$ & 1,05 & 2,27 & 1,95 & 0,78 & 0,59 & \\
\hline \multirow[t]{2}{*}{ Cáncer } & N. ${ }^{\circ}$ & 20 & 2 & 2 & 4 & 12 & 0,013 \\
\hline & $\%$ & 0,96 & 4,55 & 0,36 & 0,63 & 1,42 & \\
\hline \multirow[t]{2}{*}{ Enfermedad renal crónica en diálisis } & N. ${ }^{\circ}$ & 9 & 1 & 1 & 3 & 4 & 0,223 \\
\hline & $\%$ & 0,43 & 2,27 & 0,18 & 0,47 & 0,47 & \\
\hline \multirow[t]{2}{*}{ Cirrosis hepática } & N. ${ }^{\circ}$ & 7 & 0 & 1 & 1 & 5 & 0,418 \\
\hline & $\%$ & 0,33 & 0 & 0,18 & 0,16 & 0,59 & \\
\hline \multirow{2}{*}{ Tabaquismo } & N. ${ }^{\circ}$ & 3 & 0 & 1 & 0 & 2 & 0,674 \\
\hline & $\%$ & 0,14 & 0 & 0,18 & 0 & 0,24 & \\
\hline
\end{tabular}

La media o promedio de la duración de la hospitalización fue 8,02 días. El grupo de 50 a 64 años tuvo el mayor promedio $(p=0,000)$. De los 205 pacientes $(9,79 \%)$ que ingresaron a la $\mathrm{UCl}$, el grupo más numeroso fue el de 50 a 64 años $(p=0,000)$; la media del tiempo que un paciente estuvo en $\mathrm{UCl}$ fue de 20,40 días; y los menores de 18 años estuvieron más tiempo $(p=0,000)$. Doscientos pacientes $(9,56 \%)$ requirieron ventilación mecánica, no hubo diferencia significativa entre los grupos etarios $(p=0,942)$. Los pacientes estuvieron en ventilación mecánica un promedio de 15,26 días; los menores de 18 años mostraron el mayor promedio $(p=0,000)$.
Los pacientes que murieron fueron $843(40,28 \%)$ y la mayoría tenía de 65 años a más $(p=0,000)$. El 5,21\% (109 pacientes) de quienes recibieron ventilación mecánica fallecieron, el $54,50 \%$ de todos los pacientes con este procedimiento, sin diferencias significativas entre los grupos de edad $(p=0,191)$. Los pacientes que salieron de alta fueron $1041(49,74 \%)$, la mayoría estaba en el grupo etario de 18 a 49 años $(p=0,000)$. En el momento en que se recolectaron los datos, 209 pacientes $(9,99 \%)$ continuaban hospitalizados al momento que se recolectaron los datos (Tabla 2). 
Tabla 2. Utilización de los servicios y egresos de los pacientes hospitalizados con COVID-19, 15 de marzo al 17 de agosto del 2020

\begin{tabular}{|c|c|c|c|c|c|c|c|}
\hline \multirow[b]{2}{*}{ Característicaa } & & \multicolumn{5}{|c|}{ Grupo de edad } & \multirow{2}{*}{$\begin{array}{c}\text { Valor } \\
p\end{array}$} \\
\hline & & $\begin{array}{l}\text { Total } \\
2093\end{array}$ & $\begin{array}{l}<18 \\
(n=44)\end{array}$ & $\begin{array}{c}18-49 \\
(n=563)\end{array}$ & $\begin{array}{c}50-64 \\
(n=639)\end{array}$ & $\begin{array}{c}\Rightarrow>65 \\
(n=847)\end{array}$ & \\
\hline Media días de hospitalización & & 8,02 & 5,45 & 6,55 & 9,42 & 4,54 & 0,000 \\
\hline \multirow[t]{2}{*}{ Pacientes en $\mathrm{UCl}$} & N. ${ }^{\circ}$ & 205 & 3 & 59 & 89 & 54 & 0,000 \\
\hline & $\%$ & 9,79 & 6,81 & 10,47 & 13,92 & 6,37 & \\
\hline Media días de hospitalización en UCI & & 20,40 & 26,60 & 20,80 & 21,60 & 18,50 & 0,000 \\
\hline \multirow[t]{2}{*}{ Pacientes con ventilación mecánica } & N. ${ }^{\circ}$ & 200 & 3 & 57 & 87 & 53 & 0,942 \\
\hline & $\%$ & 9,56 & 6,82 & 10,48 & 13,62 & 6,26 & \\
\hline Media días con ventilación mecánica & & 15,26 & 17,00 & 15,26 & 16,60 & 12,94 & 0,000 \\
\hline \multirow[t]{2}{*}{ Fallecidos } & N. ${ }^{\circ}$ & 843 & 3 & 76 & 255 & 509 & 0,000 \\
\hline & $\%$ & 40,28 & 6,82 & 13,50 & 39,91 & 60,09 & \\
\hline \multirow[t]{2}{*}{ Fallecidos ventilación mecánica } & $N .^{\circ}$ & 109 & 1 & 24 & 47 & 37 & 0,191 \\
\hline & $\%$ & 5,21 & 2,27 & 4,26 & 7,36 & 4,36 & \\
\hline \multirow[t]{2}{*}{ Pacientes de alta } & N. ${ }^{\circ}$ & 1041 & 36 & 434 & 296 & 275 & 0,000 \\
\hline & $\%$ & 49,74 & 81,82 & 77,09 & 46,32 & 32,47 & \\
\hline \multirow[t]{2}{*}{ Pacientes que continúan hospitalizados } & N. ${ }^{\circ}$ & 209 & 4 & 43 & 85 & 77 & 0,044 \\
\hline & $\%$ & 9,99 & 9,99 & 7,64 & 13,30 & 9,09 & \\
\hline
\end{tabular}

\section{DISCUSIÓN}

Al analizar la información de los pacientes hospitalizados por COVID-19, encontramos que la mayoría tuvieron más de 50 años, con predominio de 65 años a más, lo que concuerda con varios estudios ${ }^{(14-17)}$. La edad media fue 58 años, lo que difiere de otras investigaciones que mostraron un promedio menor ${ }^{(18)}$, asimismo se reporta una edad mayor ${ }^{(19-22)}$, lo que muestra que las personas de 65 a más años tienen un riesgo mayor de sufrir una enfermedad grave, por lo que aumentaría la probabilidad de ser hospitalizadas ${ }^{(14,15)}$.

De todos los pacientes, los hombres formaban el grupo más numeroso, dato que coincide con otras investigaciones ${ }^{(16-19,23,24)}$, aunque difiere a una serie en la que predominaban las mujeres ${ }^{(21)}$.

Respecto a las comorbilidades, el 39,99\% de los pacientes las presentaba, lo que coincide con un reporte ${ }^{(15)}$, pero difiere de otros estudios que muestran comorbilidades en más del $50 \%$ de los pacientes ${ }^{(14,17,22-24)}$ (en el rango de $61 \%$ a $89 \%)$.

La hipertensión arterial, la diabetes mellitus y la obesidad son las principales comorbilidades de nuestra población, tal como ocurrió en otros trabajos $(15,16,19,22,25)$. Las dos primeras se presentan también en otras investigaciones en las que se relacionan con una mayor gravedad $(14,15,18,19,24,26)$. Algunos autores no consideran que la obesidad puede agravar los casos de COVID-19 ${ }^{(14,15)}$, aunque un estudio reciente ha determinado que la obesidad sí está relacionada con la severidad del cuadro ${ }^{(27)}$. Asimismo, se refiere que los grupos minoritarios raciales y étnicos tienen un riesgo más alto de tener enfermedad grave por COVID-19, ya que la prevalencia de dolencias previas es mayor en ellos ${ }^{(16)}$.

En los pacientes de más de 65 años, la comorbilidad más frecuente fue la hipertensión arterial seguida de la diabetes mellitus, al igual que en estudios anteriores ${ }^{(16,22)}$. La obesidad se observó en las personas de 18 a 49 años, al igual que en un informe previo ${ }^{(16)}$. En esta investigación, la frecuencia de enfermedad cardiovascular, asma, cáncer, enfermedad renal crónica, cirrosis hepática y tabaquismo fue menor del 1,10\%; sin embargo, en Inglaterra, la enfermedad cardiovascular apareció en el $31 \%$ de los casos y la enfermedad renal crónica, en el $16 \%{ }^{(23)}$.

Las características de los grupos de edad, el sexo y las comorbilidades descritas en los pacientes con COVID-19 pueden estar relacionadas con la prevalencia de las enfermedades, tal como se menciona en algunos reportes ${ }^{(14,28)}$.

La media de la duración de hospitalización fue 8,02 días, tiempo que es menor al de un estudio que refiere un periodo de 10,40 días ${ }^{(22)}$. El 9,79\% de los pacientes internados fueron atendidos en la Unidad de Cuidados Intensivos; en distintas series, este tipo de atención varía de $3,40 \%$ al $24 \%$ (15,19,21,23); la mayor cantidad de pacientes estaban en el grupo de 50 a 64 años, y en otros estudios, en el grupo de 65 años a más ${ }^{(16)}$. 
La mortalidad fue $40,28 \%$, similar a lo reportado ${ }^{(20)}$, aunque es alta respecto a otros estudios ${ }^{(16,21-23)}$ en los que la mortalidad fue del $17 \%$ al $26 \%$; la mayoría de las muertes ocurrió en los pacientes de 65 años a más, similar a una investigación europea (21). El 54,50\% $(109 / 200)$ de los pacientes que recibieron ventilación mecánica fallecieron, sin diferencias significativas entre los grupos de edad, este resultado es alto respecto a otras publicaciones ${ }^{(16,17)}$, pero menor que una serie de casos de China ${ }^{(18)}$. Aproximadamente, la mitad de los pacientes fueron dados de alta, lo que coincide con un estudio previo ${ }^{(23)}$.

Esta investigación, al ser retrospectiva, tiene como limitaciones que los datos provienen de una muestra censal y que los pacientes no fueron seguidos desde que fueron hospitalizados, pues la información empleada estaba registrada en una base de datos.

De los resultados obtenidos, recomendamos mejorar el manejo de las comorbilidades e implementar el seguimiento clínico para disminuir las hospitalizaciones y continuar promoviendo las medidas que disminuyen la propagación de la infección, por ejemplo, quedarse en casa, usar las mascarillas, el distanciamiento social, la ventilación del ambiente e higiene de manos, para proteger a los grupos considerados de riesgo, así como a los que no tienen tal condición ${ }^{(16,17,26,29,30)}$.

Contribuciones de los autores: Marco Antonio Alfaro Angulo: diseño del estudio, recolección de los datos, revisión de la literatura, análisis e interpretación de resultados y redacción del artículo. Mónica Rosaura Rivero Villegas: recolección de los datos, revisión de la literatura, análisis de resultados y redacción del artículo. Víctor Andrés Sánchez Reyna: revisión de la literatura, análisis e interpretación de resultados.

Fuentes de financiamiento: Este artículo ha sido financiado por los autores.

Conflicto de intereses: Los autores declaran no tener ningún conflicto de intereses.

\section{REFERENCIAS BIBLIOGRÁFICAS}

1. Ribao H. Wuhan Municipal Health Commission announces pneumonia epidemic [Internet]. 2019. Disponible en: https://crofsblogs. typepad.com/h5n1/2019/12/wuhan-municipal-health-commissionannounces-pneumonia-epidemic.html

2. XinhuaNet. New-type coronavirus causes pneumonia in Wuhan: expert [Internet]. 2020. Disponible en: http://www.xinhuanet.com/ english/2020-01/09/c_138690570.htm

3. Johns Hopkings University Medicine. Coronavirus Resource Center [Internet]. 2021. Disponible en: https://coronavirus.jhu.edu/map.html

4. Ministerio de Salud. Sala Situacional COVID-19 Perú [Internet]. MINSA: Perú; 2021. Disponible en: https://covid19.minsa.gob.pe/ sala_situacional.asp

5. Ministerio de Salud. Red Asistencial La Libertad-EsSalud. Sala de Situación COVID-19 [Internet]. MINSA: Perú; 2021. Disponible en: https://www.gob.pe/red-asistencial-la-libertad

6. Guan W-J, Ni Z-Y, Hu Y, Liang W-H, Ou C-Q, He J-X, et al. Clinical characteristics of Coronavirus Disease 2019 in China. N Engl J Med 2020; 382: 1708-20.

7. Fuk-Woo Chan J, Yuan S, Kok K-H, Kai-Wang To K, Chu H, Yang J, et al. A familial cluster of pneumonia associated with the 2019 novel coronavirus indicating person-to person transmission: a study of a family cluster. Lancet. 2020; 395(10223): 514-23.

8. Whan Z. The Coronavirus Prevention Hanbook. China: Eskihorse Publishing; 2020. p. 126.

9. Wu Z, McGoogan JM. Characteristics of and important lessons from the Coronavirus Disease 2019 (COVID-19) outbreak in China: summary of a report of 72314 cases from the Chinese Center for Disease Control and Prevention. JAMA. 2020; 323(13): 1239-42.

10. Ministerio de Salud. Alerta Epidemiológica Código: AE-15-2020 Alerta Epidemiológica ante la transmisión de COVID-19 en el Perú [Internet]. MINSA: Perú; 2020. Disponible en: https://www.dge.gob. pe/portal/docs/alertas/2020/AE015.pdf

11. World Health Organization. Report of the WHO-China Joint Mission on Coronavirus Disease 2019 (COVID-19) [Internet]. WHO; 2020. Disponible en: https://www.who.int/docs/default-source/ coronaviruse/who-china-joint-mission-on-covid-19-final-report.pdf

12. Wang D, Hu B, Hu C, Zhu F, Liu X, Zhang J, et al. Clinical characteristics of 138 hospitalized patients with 2019 Novel Coronavirus-Infected Pneumonia in Wuhan, China. JAMA. 2020; 323(11): 1061-9.

13. Tinku J, Mohammed AM. International pulmonologist's consensus on Covid-19. 2020. p. 43.

14. Garg S, Kim L, Whitaker M, O'Halloran A, Cummings C, Holstein R, et al. Hospitalization rates and characteristics of patients hospitalized with Laboratory-confirmed Coronavirus Disease 2019 - COVID-NET, 14 States, March 1-30, 2020. MMWR Morb Mortal Wkly Rep. 2020; 69(15): 458-64.

15. Petrilli CM, Jones SA, Yang J, Rajagopalan H, O'Donnell L, Chernyak $Y$, et al. Factor associated with hospital admission and critical illness among 5279 people with coronavirus disease 2019 in New York City: prospective cohort study. BMJ. 2020; 369: m1966.

16. Gold JAW, Wong KK, Szablewski CM, Patel PR, Rossow J, da Silva $J$, et al. Characteristics and clinical outcomes of adult patients hospitalized with COVID-19 - Georgia, March 2020. MMWR Morb Mortal Wkly Rep. 2020; 69(18): 545-50.

17. Killerby ME, Link-Gelles R, Haight SC, Schrodt CA, England L, Gomes $\mathrm{J}$, et al. Characteristics associated with hospitalization among patients with COVID-19 - Metropolitan Atlanta, Georgia, March-April 2020. MMWR Morb Mortal Wkly Rep. 2020; 69(25): 790-4.

18. Yang X, Yu Y, Xu J, Shu H, Xia J, Liu H, et al. Clinical course and outcomes of critically ill patients with SARS-CoV-2 pneumonia in Wuhan, China: a single-centered, retrospective, observational study. Lancet Respir Med. 2020; 8(5): 475-81.

19. Richardson S, Hirsch JS, Narasimhan M, Crawford JM, McGinn T, Davidson KW, et al. Presenting characteristics, comorbidities, and outcomes among 5700 patients hospitalized with COVID-19 in the New York City Area. JAMA. 2020; 323(20): 2052-9.

20. Ranzani OT, Bastos LSL, Gelli JGM, Marchesi JF, Baião F, Hamacher $\mathrm{S}$, et al. Characterisation of the first 250000 hospital admissions for COVID-19 in Brazil: a retrospective analysis of nationwide data. Lancet Respir Med. 2021; 9(4): 407-18.

21. Borobia AM, Carcas AJ, Arnalich F, Álvarez-Sala R, Monserrat J, Quintana M, et al. A cohort of patients with COVID-19 in a major teaching hospital in Europe. medxRiv. 2020; 9(6): 1-10.

22. Casas-Rojo JM, Antón-Santos JM, Millán-Nuñez-Cortés J, 
Lumbreras-Bermejo C, Ramos-Rincón JM, Roy-Vallejo E, et al. Características clínicas de los pacientes hospitalizados con COVID-19 en España: resultados del Registro SEMI-COVID-19. Rev Clin Esp. 2020; 220(8): 480-94.

23. Docherty $A B$, Harrison EM, Green CA, Hardwick HE, Pius R, Norman L, et al. Feature of 20133 UK patients in hospital with COVID-19 using the ISARIC WHO Clinical Characterisation Protocol: prospective observational cohort study. BMJ. 2020; 369: m1985.

24. Acosta G, Escobar G, Bernaola G, Alfaro J, Taype W, Marcos C, et al. Caracterización de pacientes con COVID-19 grave atendidos en un hospital de referencia nacional del Perú. Rev Perú Med Exp Salud Publica. 2020; 37(2): 253-8.

25. Treviño JA. Demografía, comorbilidad y condiciones médicas de los pacientes hospitalizados por Covid-19 en México. MARLAS. 2020; $4(1) ; 49-70$.

26. Centers for Disease Control and Prevention. People with certain medical conditions [Internet]. CDC: USA; 2021. Disponible en: https: / / www.cdc.gov/coronavirus/2019-ncov/need-extraprecautions/people-with-medical-conditions.html?CDC_AA refVal=https\%3A\%2F\%2Fwww.cdc.gov\%2Fcoronavirus\%2F2019ncov\%2Fneed-extra-precautions\%2Fgroups-at-higher-risk.html\#

27. Kompaniyets L, Goodman AB, Belay B, Freedman DS, Sucosky MS, Lange $J$, et al. Body Mass Index and Risk for COVID-19-Related Hospitalization, Intensive Care Unit Admission, Invasive Mechanical Ventilation, and Death- United States, March-December 2020. MMWR Morb Mortal Wkly Rep. 2021; 70(10): 355-61.

28. Centers for Disease Control and Prevention. Hypertension Prevalence and control among adults: United States, 2015-2016. NCHS Data Brief № 189 [Internet]. CDC: USA; 2017. Disponible en: https://www.cdc. gov/nchs/products/databriefs/db289.html

29. Centers for Disease Control and Prevention. Coronavirus Disease 2019 (COVID-19): what you can do [Internet]. CDC: USA; 2020. Disponible en: https://www.cdc.gov/coronavirus/2019-ncov/needextra-precautions/what-you-can-do.html

30. Centers for Disease Control and Prevention Coronavirus Disease 2019 (COVID-19): how to protect yourself and others [Internet]. CDC: USA; 2021. Disponible en: https://www.cdc.gov/coronavirus/2019-ncov/ prevent-getting-sick/prevention.html
Correspondencia:

Marco Antonio Alfaro Angulo

Dirección: Urb. Juan Pablo II, 2da. Etapa - E1, Trujillo. La Libertad, Perú.

Teléfono: +51924730989

Correo electrónico: marcoalfaroa@yahoo.com.mx

Recibido: 24 de abril de 2021

Evaluado: 26 de mayo de 2021

Aprobado: 14 de junio de 2021

(c) La revista. Publicado por Universidad de San Martín de Porres, Perú. (c) ${ }_{\text {BY }}$ Licencia de Creative Commons Artículo en acceso abierto bajo términos de Licencia Creative Commons Atribución 4.0 Internacional. (http://creativecommons.org/licenses/by/4.0/)

\section{ORCID iDs}

Marco Antonio Alfaro Angulo (1) https: / / orcid.org/0000-0002-6105-4649 Mónica Rosaura Rivero Villegas i http://orcid.org/0000-0001-9746-2145

Víctor Andrés Sánchez Reyna ㄴ https://orcid.org/0000-0002-8998-4119 